\title{
A Smart System for Health Caregiver Based on IoMT: Toward Tele-Health Caregiving
}

\author{
https://doi.org/10.3991/ijoe.v17i07.22525
}

\author{
Azmi Shawkat Abdulbaqi $(\bowtie)$ \\ University of Anbar, Al Anbar, Iraq \\ azmi msceuoanbar.edu.iq
}

Ahmed J.Obaid

University of Kufa, Najaf, Iraq

Sundos Abdulameer Hmeed Alazawi

Al Mustansiriyah University, Baghdad, Iraq

\begin{abstract}
This manuscript introduces the designing and emulation of a wireless, intelligent health surveillance device based on the internet of medical things, with GSM alert technology by health caregivers. In hospitals or medical centers where the body temperature of the patient needs to be constantly checked or other vital signs have to be checked, this is normally achieved by a specialist or another paramedicine personnel by continuously checking the temperature, pulse rate and keeping records of these arguments. This approach is really repetitive and can be very tiring, especially in medical centers crowded with patients. The main system task is to surveillance the patient's body pulse rate and the patient's body temperature and sending the information to the specialists wirelessly via the liquid crystal display. The transmitting unit of this suggested system continuously surveillance the patient's body temperature, as well as the heart rate with digital sensors, and ultimately, displays the outcomes on the liquid crystal display transmitter to present to the specialists. The receiver device is placed in the specialist chamber for continuous wireless surveillance of the patient's body temperature and heart rate. At the receiver end where the specialist will be, an alarm is often triggered and triggered When the patient's heart rate or temperature is below/above the normal human threshold of $37^{\circ} \mathrm{C}$ and 70 beats/min (bpm), then, send an SMS to the specialist if he or she is absent as a means of continuous update. The suggested framework was implemented utilizing Proteus Tools and programs, composed in Embedded C. The obtained outcomes indicate an effective way to relay details on duty to the specialists for urgent patient care.
\end{abstract}

Keywords - Internet of Medical Things (IoMTs), Galileo Microcontroller, Arduino UNO Type WEMOS D1-ESP 8266 (WEMOS-Uno), GSM technology 


\section{Introduction}

Recently, significant advances also brought modern medical technologies. Most healthcare centers and hospitals are seeking to make more alert and preventive treatments accessible and to maintain lasting treatments that are successful. A professional Smart Surveillance system for Patients requires this successful treatment [1].

The patient management system is a highly advanced technology which regulates and tracks the condition of similar criteria of human parameters. Some of our parameters for human health, such as temperature, respiration, blood flow, pulse rate, etc., require continuous surveillance and updating to duty specialists.

Mobile computing defines a modern wave of mobile devices that now predominate in daily life. Handheld mobile phone and a variety of embedded systems make the information and internet connectivity easy to access from anywhere at any time. The aim of mobile health caregiver is to offer healthcare services to everyone anywhere at any time, addressing location, time and money constraints. While the prominent presenting systems available for surveillance of the vital signs of the patient, these systems require that the sensors be positioned at bedside surveillance or PCs, and that the patient be confined to his bed [2]. This suggested method does not enable patients to be confined to their beds, but requires them to move around at a defined distance, however. This is because the prospect of gathering data beyond this context is unfeasible.

Wireless sensor network (WSN) combination, proven Radio Frequency Identification (RFID) and vital signs tracking equipment is utilized to continuously surveillance vital signs while maintaining position tracking of the users. The utilize of wireless technologies enables the network to be mounted in our homes and to be connected to various hospitals. Waves of radio frequencies can penetrate walls and materials, sending information about the vital signs and positions to a central tracking computer server at the end of the patients via a miniature transmitter network. Wireless technology's advantages are already evident: portability, flexibility, ease of deployment and cost-effectiveness [3]. The common interest of governments, business, and academic community is one of the main contributing factors to that. Indeed, the findings and concepts outlined in this paper should promote and drive work on machine learning and Artificial Intelligence(AI) systems for informed decision-making in healthcare technology and delivery. The rest of the manuscript is organized as follows: Review of literatures has been listed in Section 2. Section 3, introduced the research objectives. Section 4 implemented program methodology. Hardware framework, defined in Section 5. Section 6 discusses conclusions and discussions.

\section{Review of Literature}

Below, some of the related works with objective of the manuscript, these works are:

In [4], Abdulbaqi A.S., Saif Al-din M. Najim, and Ismail Yusuf Panessai. Introduced a full method for patient surveillance real-time that surveil patients in multiple environments. This system is consists of WEMOS-UNO connect with WSN directly on the patient body by many electrodes and finally connect directly with PC to implement a 
complete connection to the specialist online. In [5], Fatusi et.al considered symptom uncertainty and causal associations between several diseases and symptoms to obtain a detailed estimate with some degree of confidence. Implementing a processor that collects sensor signals for patient safety. In [6], Shihab A. Hameed et al (2008) developed a framework model that utilizes open source software for medical records centralized, which can be accessed and updated by specialists utilizing any internet browser. In [7], A.I. Heernande et al. proposed a full real-time framework for the processing, transmission, storage, and visualization of electrocardiogram (ECG) for non-clinical applications. In [8], Raised, M.F.A., Woodware, B., focused on designing the processor, which extracts patient signals from sensors. It then passes on digital data over a Bluetooth connection to a mobile phone utilizing the "General Packet Radio Service" (GPRS). The main contributions of this work are the following: (I) using new generation of Microcontroller called Arduino UNO Type (model WEMOS D1-ESP 8266), (ii) Explored further research works by the research community.

\section{$3 \quad$ Research Objective}

The novel healthcare system is a bright spot in the medical world, as it permits doctor-patient contact by examining the relationship between the body's biological conditions. The aim of the research is to: (1) Design a reliable prototype that surveillance various vital parameters of the human body and sends data to specialists based on IoMT as a step towards Telemedicine; and sends alert in cases of decreased performance of vital functions of the body. (2) To improve the patient's experience of knowing the health conditions of health caregivers.

\section{$4 \quad$ Research Methodology}

The Wireless Patient Intelligent Health Surveillance System (WPIHSS) is designed in two segments namely: the transmitter segment in the area of the patient and the recipient segment in the area of the specialist. To actualize these design research objectives, the block diagram of the wireless health intelligent surveillance system utilized is shown in Fig.1. 


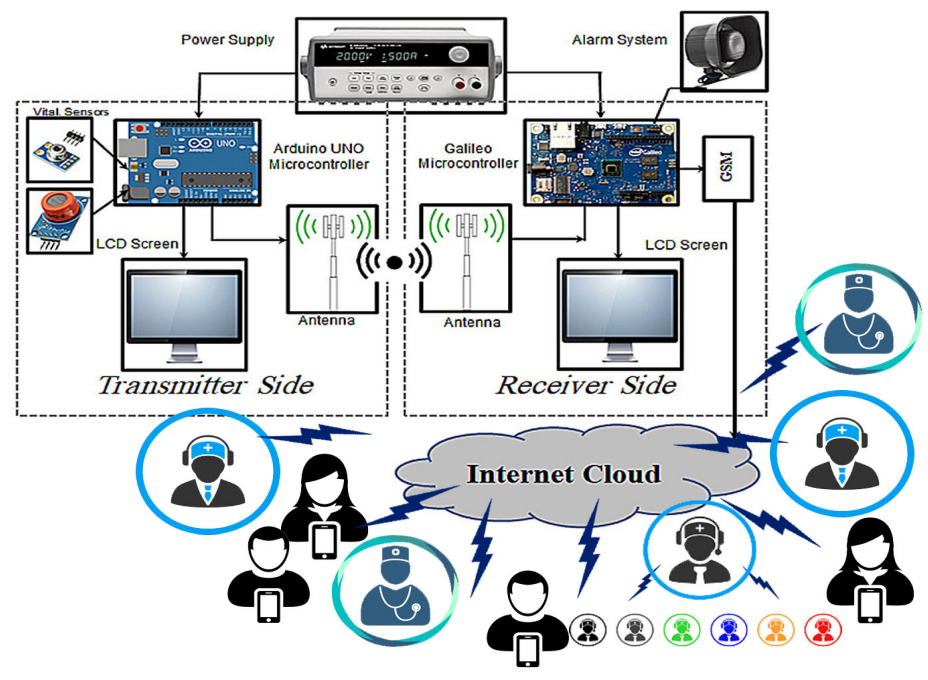

Fig. 1. IoMT Based Health Caregiver Smart Surveillance System Schematic

\subsection{Surveillance system based transmitter}

Consistent with the presented diagram in Fig. 2 below, a proposed sending system is designed at the patient's location. The transmitting section consists of power supply, temperature sensor, pulse sensor, WEMOS-UNO (Microcontroller), Liquid Crystal Display (LCD) and RF antenna, which is the RF link transmitter. Power Supply is a device that replaces the entire WPIHSS network with electric power. The pulse sensor is built in the transmitter to provide digital heart beat output when it touches with the finger. The beat LED flashes at one with each heartbeat while the heart beat detector is operating. The output of heartbeat can be connected to Microcontroller to measure the bpm rate through program code directly. It operates on the theory of light regulation of blood flow at any pulse through the finger. The temperature sensor is attached to the health patient whose temperature is to be measured. Its values are expressed in millivolts $(\mathrm{mV})$, which is sent to the WEMOS-UNO Microcontroller. This is received as analog signals between 0-1024 and mapped to $5 \mathrm{~V}$ through written program codes. The value is scaled by the WEMOS-UNO and converted to a digital standard which is sent to the transmitter RF antenna and further displayed on transmitter's LCD. Such parameters are sent via the RF link transmitter antenna to the receiver section of the specialist 's side where the data is read by the health worker or the specialist in charge [9]. 


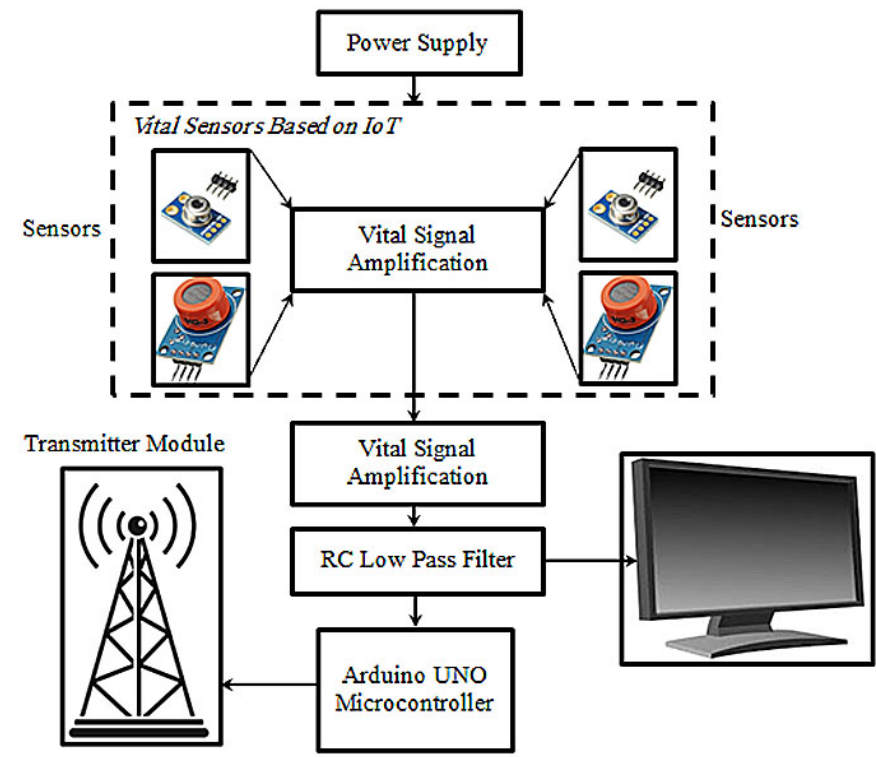

Fig. 2. Infrastructure of Transmission Side

The pulse sensor and the temperature sensors are connected to the A0 and A1 pins on the WEMOS-UNO board. The LCD pins are plugged into the pins 2, 3, 4 and 12 , $10,9,8$ pins on the WEMOS-UNO board. Connects a rechargeable battery to the WEMOS-UNO power port.

\subsection{Surveillance system based transmitter flowchart}

The flowchart in the Fig. 3 below shows the transmitter section that was utilized to realize the program part which controls the transmitter section of the system. When properly coded, the microcontroller input and output pins become initiated. The initiation triggers the microcontroller to check input values from the temperature and pulse sensors [10]. Since the microcontroller has been programmed in such a way that it can map out the voltage values from the sensors, and convert to digital form, it further sends this converted values to the transmitter. The transmitter on receiving these values sends output outcomes to the LCD for display. There will exist a delay time in pre-defined seconds, before another reading will be taken, after which one cycle of the program is ended. This process continues checking for temperature and pulse rate and relating the value to the Microcontroller for further processing [11]. 


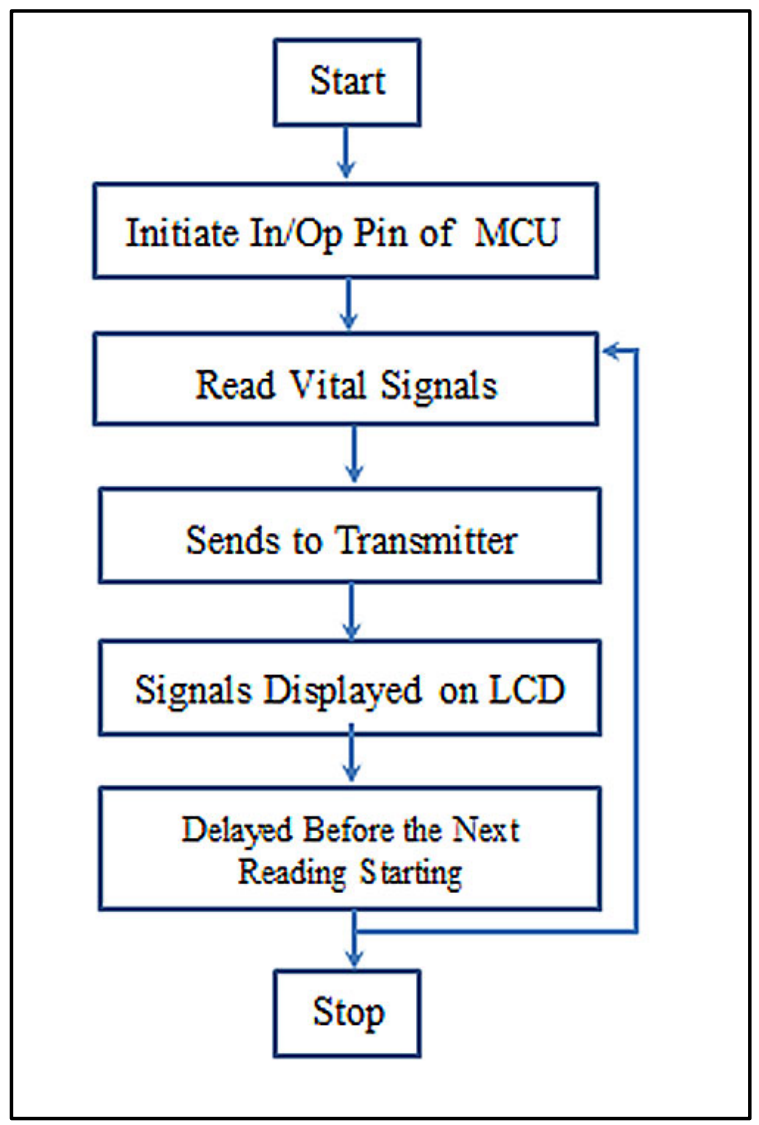

Fig. 3. Flowchart of the Transmitter Side

\subsection{Surveillance system based receiver section}

The signal from the transmitting section is received by the RF link receiver antenna. It works as a wireless data link, establishing connection between the transmitting and receiving sections of the system. The received signal is sent to the Intel Galileo which processes the data and sends it to the specialists LCD for processed parameter display [12]. Also the processed parameters are sent to the specialist's phone through GSM shield and to the internet cloud for storing and sharing. The GSM shield acts as a GSM modem that sends SMS to the specialist's phone once the value of the patient's health data goes above a certain threshold. The Fig. 4 below displays the block diagram of the receiver section. The Internet cloud stores the patient's data in a database and can be assessed online through internet enabled computer anywhere. The LCD placed in the specialists office displays outcome of the patient's parameters within a certain programmed period of time [13]. 


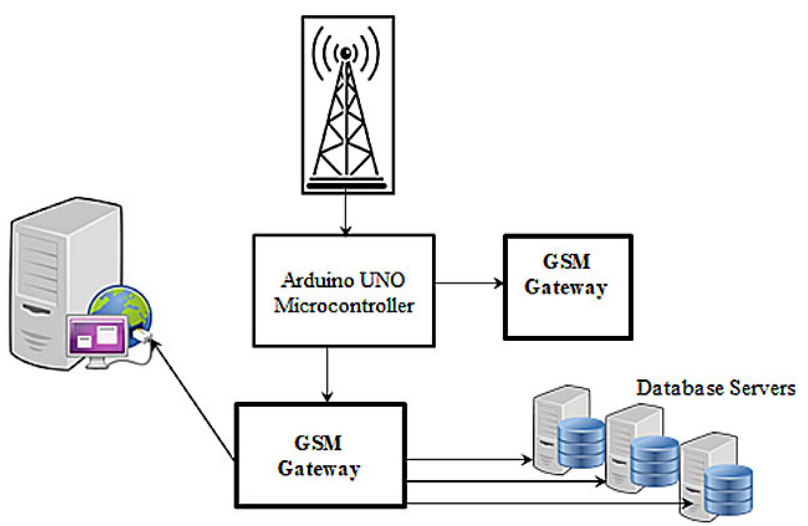

Fig. 4. Infrastructure of Receiver Side

The Intel Galileo board has microcontroller that processes the received signals from the RF antenna of the transmitter section. The LCD is connected to the pins 4, 5, 6, 7, 8 of the GSM shield, which is mounted on top of the Intel board and connected to all the pins of the Intel board. The RF link receiver is connected to the pins A0-A5 of the GSM shield and SIM card inserted in the SIM slot of the GSM shield, as this would be utilized as the mobile link in other to send SMS to the specialists phone [14].

\subsection{Surveillance system based receiver flow chart}

The flow chart in the Fig. 5 below shows the receiver section that was utilized to realize the program part which controls the receiver section of the system. The system starts by checking the Microcontroller input and output pins through the antenna. If the Microcontroller receives a value from the transmitter antenna, it will analyze and compare with the pre-defined programmed value. Again, if it does not receive any value from the transmitter, it will continue checking [15]. On the other hand, if the value received from the transmitter is less than or equal to the threshold value, then the value will be displayed as an output on the LCD display unit to be seen by the specialists or health worker. An SMS alert will be sent to the specialists if the value supersedes or is flagged as an emergency value. Also, an alarm system will be triggered to alert the specialists in charge of the health patient. The displayed information would last for at least 20 minutes before the system removes the data in its display unit for another display. This process continues displaying of the health patient's parameters for specialists notification and quick action [16]. 


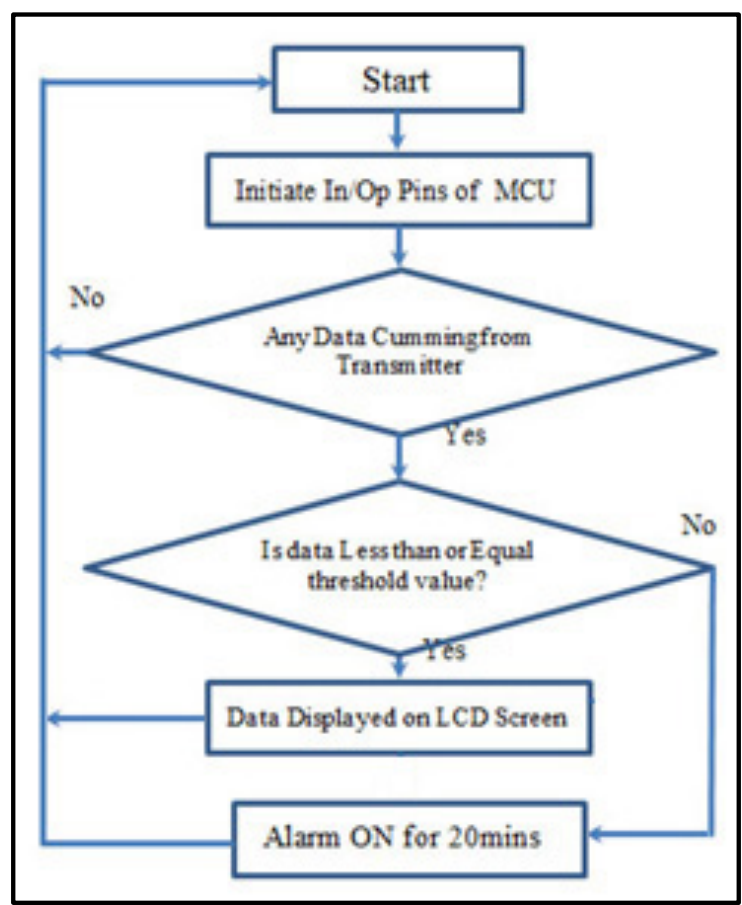

Fig. 5. Flowchart of the Receiver Side

\subsection{Surveillance system based operations and circuit}

WPIHSS operates utilizing $+5 \mathrm{v}$ supply voltage from the external regulated battery source or direct plug to the USB port of the Laptop. When properly set and powered $\mathrm{ON}$, the transmitter section and receiver section are initialized for operations through the embedded $\mathrm{C}$ language. At the transmitter section, the Pulse sensor is designed to be worn by the patient and temperature sensor placed anywhere on the body of the patient which capture patient body temperature as well as the pulse rate. Both are sent to the WEMOS-UNO Microcontroller which processes the data, display the parameters on the patient's LCD and send the same parameters through RF antenna of the transmitter to the specialists end wirelessly [17][8]. At the receiver section which is in specialists office as well as mobile phone; the receiver RF antenna receives the transmitted signal with the same Radio frequency and sends to the Intel Galileo processor which processes the received data, activate alarm for 20 seconds to attract specialists attention. Then display the processed data on the LCD unit in the specialists office. Also SMS message of the same parameters are sent utilizing the GSM shield through SIM card to specialists phone in case he or she is not in the hospital vicinity for urgent attention to the patient [19][20]. 


\subsection{Surveillance system based programming language}

The programming Language utilized to develop the system codes that control the designed system is WEMOS-UNO C language. The codes follow the flow chart procedures as shown in the following codes[21]. Below, Part of the C-Code for the vital signs for Transmission-Side in the embedded systems.

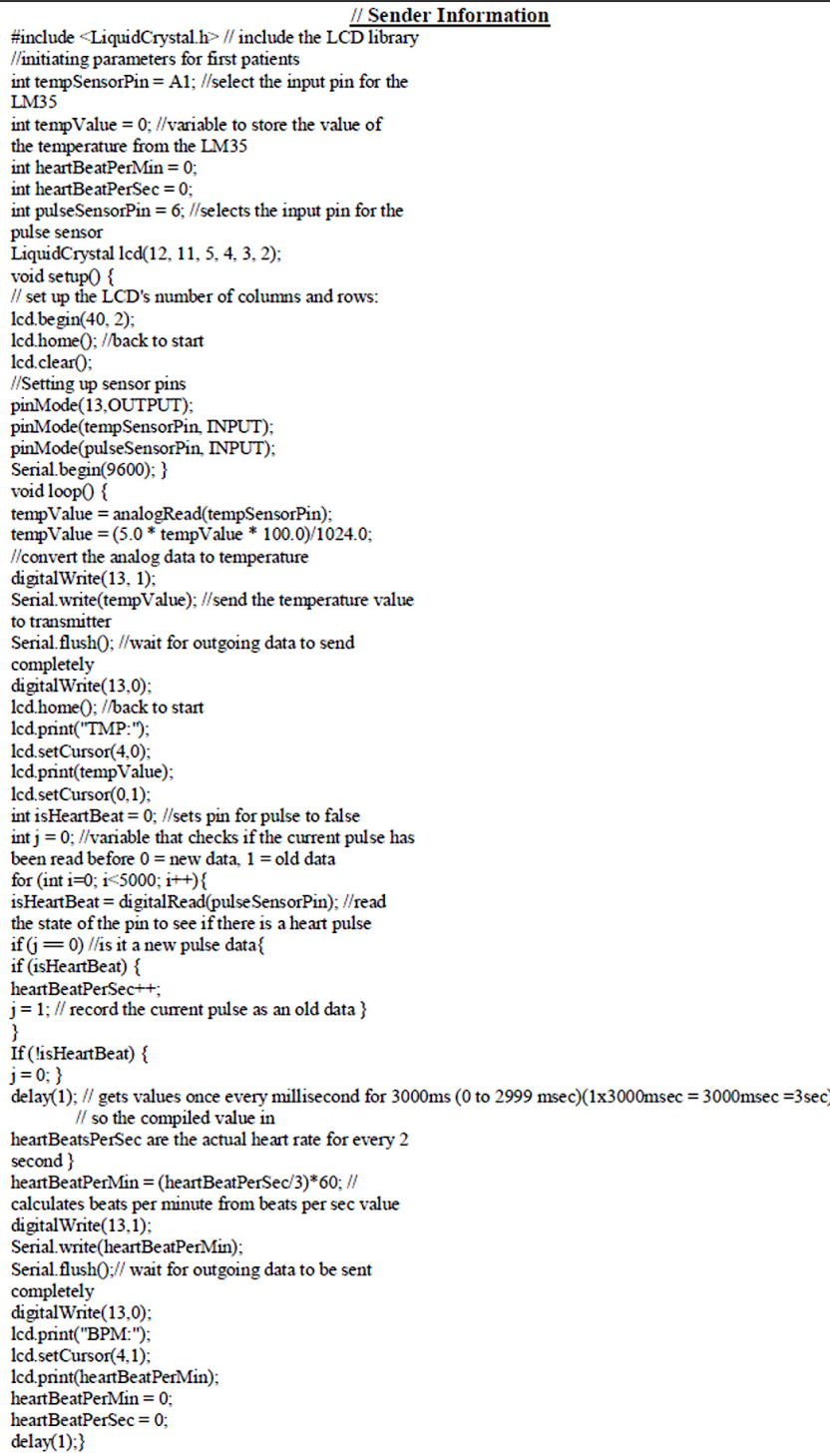


Below, Part of the C-Code for the vital signs for Receiver-Side in the embedded systems

\section{// Receiver Information}

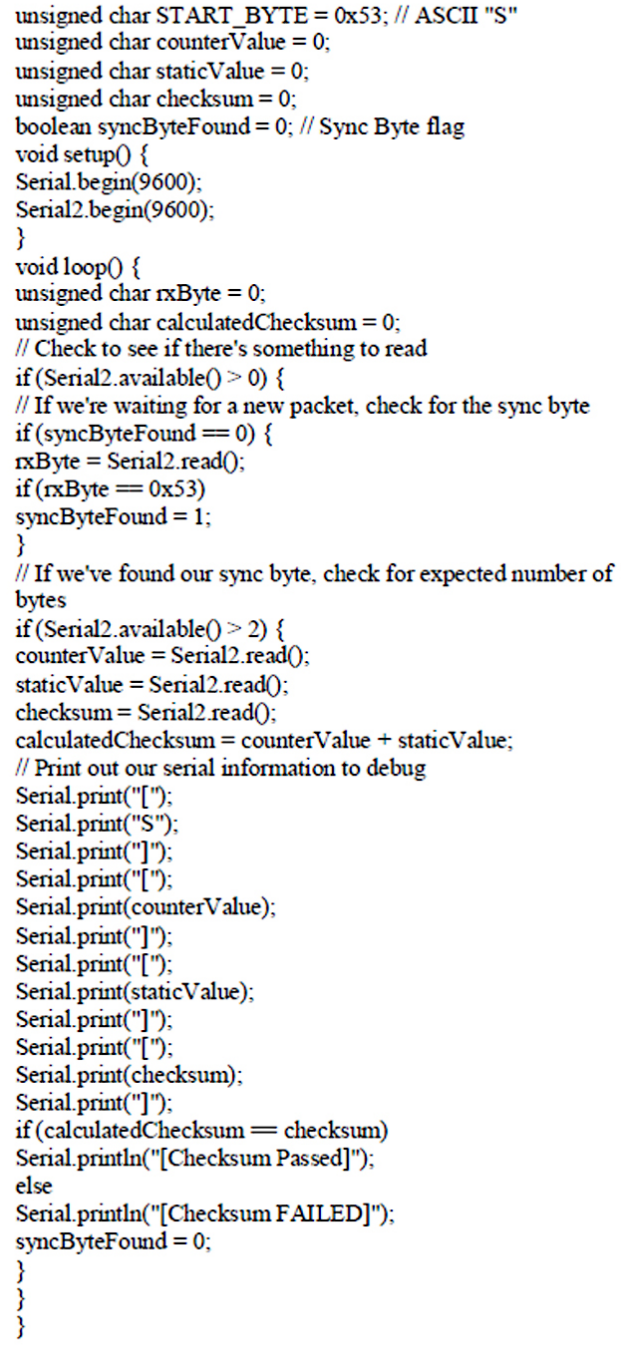

The receiver section embedded codes were not included but the reader can request through email and it will be sent. 


\section{System Components}

\subsection{Hardware parts}

The Bio-Medical Sensors that have been utilized and tested in this suggested system consist of: i) GS is a Glucometer-Sensor for measuring blood glucose. (ii) DBpS is a Digital BP Sensor to track the circulatory system; (iii) POS is a Pulse Oxymeter Sensor for measuring blood oxygen; iv) EMGS is an electromyography sensor for muscle activity assessment, (v) TS is Temperature sensor for body temperature measurement [22].

\subsection{Software parts}

Tera Term is the perfect app utilized to manage WEMOS-UNO systems. In order to obtain a correct and clear data from various biomedical sensors, WEMOS-UNO is utilized for WEMOS-UNO programming [23][24]. Android is utilized for data processing purposes and to transfer these data to a smartphone as it is utilized with iOS as platforms for displaying vital tag data. The Android and iOS configurations are set independently. Tera term is utilized for RN-XV Wifly configuration. An XBee USB adapter is connected to the RN-XV Wifly to create a serial connection during the configuration. [25][26].

\section{$6 \quad$ Outcomes and Discussions}

The simulation of the WPIHSS system design was done utilizing PROTEUS software. The system was divided into two parts as earlier mentioned; both were integrated to achieve the design objectives and communication purposes. The transmitter and receiver sections outcome obtained at different conditions are explained below.

\subsection{Patient side (client) based transmitter section}

The transmitter part outcomes based on low conditions: The outcome obtained in simulating designed transmitter circuit at low temperature and pulse rate are shown in Fig 6 below. At $33^{\circ} \mathrm{C}$, the human body temperature is at low and is said to be critical, and at $60 \mathrm{bpm}$ the pulse rate is also at low. The values are obtained from the temperature sensor and pulse rate sensor that are placed at the transmitter section. These signals are sent in volts to the WEMOS-UNO in other to be read and convert into digital forms. The outcome displayed on the LCD unit shows the signal values received from the temperature and pulse sensors in real time during the testing period. 


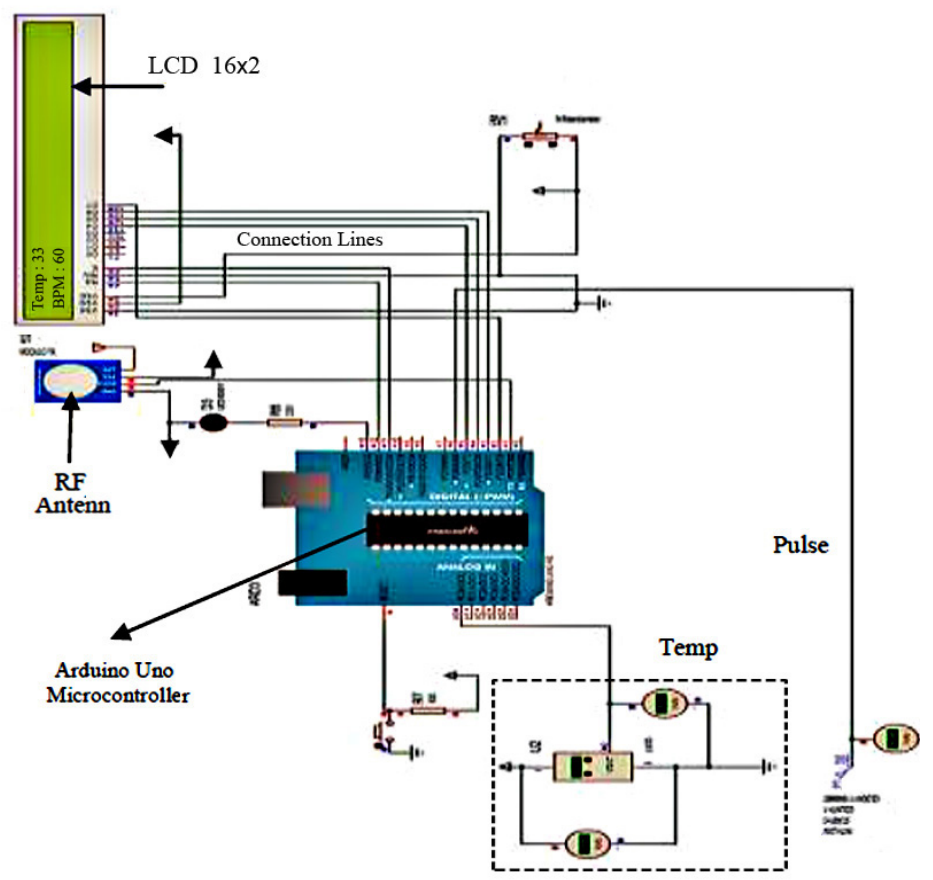

Fig. 6. The Outcomes of Transmitter Side at Low Condition

The transmitter part outcomes based on normal conditions: This shows a temperature reading at $37^{\circ} \mathrm{C}$, which is the normal human body temperature. This value is obtained from the temperature sensor which is placed at the transmitter unit that transmits the signal to the WEMOS-Uno. The WEMOS-UNO converts the signal and further sent to the receiver through the RF antenna unit. The temperature value is displayed on the LCD at the transmitter section.

The transmitter part outcomes based on extreme / high conditions: The values displayed on the transmitter unit LCD are $39^{\circ} \mathrm{C}$ against bpm 120 . The temperature value is high for human being because any value above $37^{\circ} \mathrm{C}$ is flagged as extreme and serves as a symptom of high fever. Also the bpm value is recorded as 120 , which is also a high pulse rate as any value above 100 is deduced as being extreme, irregular and critical. These values are displayed on the transmitter LCD, and transmitted to the receiver LCD, which is placed in the specialist's office. The receiver part outcomes obtained at different conditions are shown below.

\subsection{Receiver side based on server section (specialist side)}

The receiver part outcomes based on low conditions: The outcome obtained from the simulating of the receiver circuit at low condition is shown in Fig 7 below. This shows a temperature reading at $33^{\circ} \mathrm{C}$, which is a low human body temperature. The outcome is transmitted to the receiver unit from the transmitting section. As the 
receiving unit will be at the specialists office, the display unit (LCD) at the specialists office will display the temperature and pulse rate of the patient. An alarm is incorporated in the circuit to trigger at any temperature below $34^{\circ} \mathrm{C}$, and also at a pulse rate of $60 \mathrm{bpm}$ and below. This alarm will continue for 20 minutes until it is reset by the specialists or health worker in charge. An SMS containing the details of the patient's temperature and pulse rate parameters will be sent to the specialists phone for proper recording and acknowledgement, especially if the specialists is not physically present at that moment.

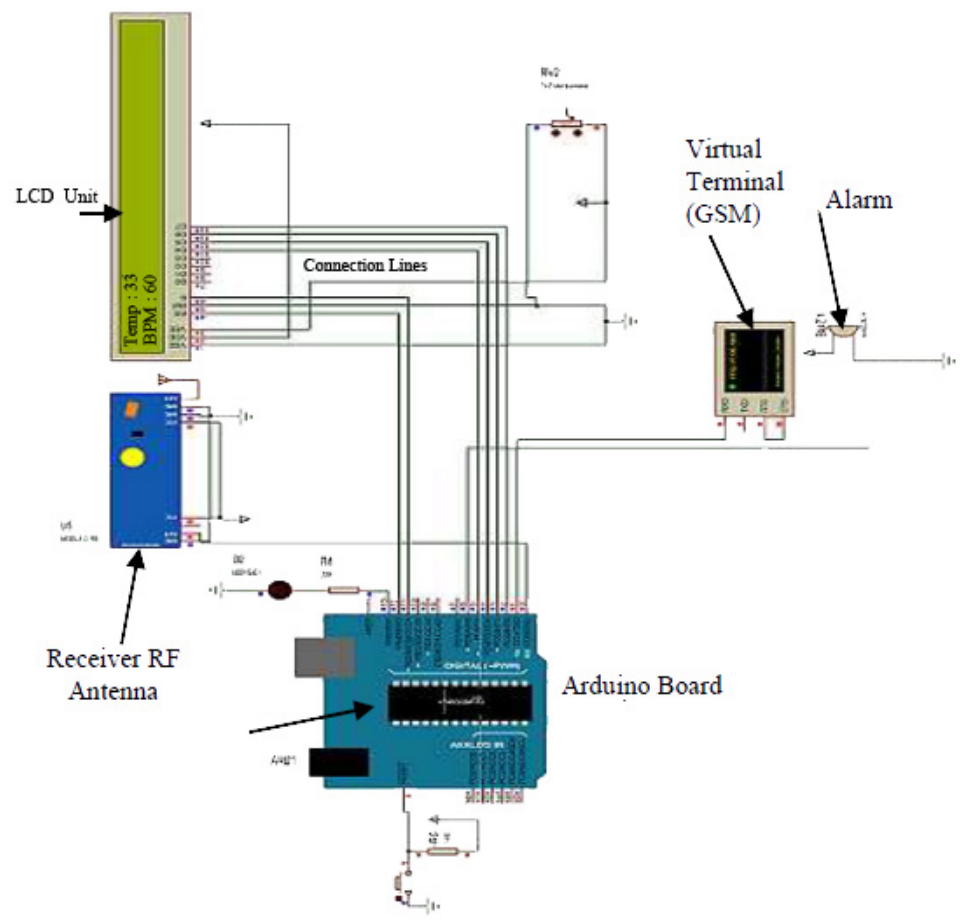

Fig. 7. The Outcomes of Receiver Side at Low Condition

The receiver part outcomes based normal conditions: This shows a temperature reading at $37^{\circ} \mathrm{C}$, which is the normal human body temperature. This value was sent from the transmitter section through RF antenna to the receiver RF antenna. The antenna sends the signal to the WEMOS-UNO Intel Galileo which processes and displays it on the specialists LCD.

The receiver part outcomes based on high conditions: The RF link transmitter receives the signal from the transmitter unit, sends to WEMOS-UNO (where the processing is done) and the outcome is displayed on the LCD in the specialists office. An alarm is activated when signals of high temperature and pulse rate parameters are received. The alarm is programmed to continue for 20 minutes until the system is reset by the specialists or health worker. 


\subsection{SMS technology}

The GSM shield card is inserted on the WEMOS-UNO board expansion slot provided and configured for SMS communication to Mobile phone through AT program codes. The network SIM card slot is found on the GSM shield board where any network SIM card can be slotted for utilize. The WEMOS-UNO GSM Shield as illustrated in Fig. 8 links WEMOS-UNO to the web utilizing wireless GPRS. This module is connected under the WEMOS-UNO frame, install an operator's SIM card with GPRS coverage and follow a few basic instructions to start surveilling your environment over the Internet

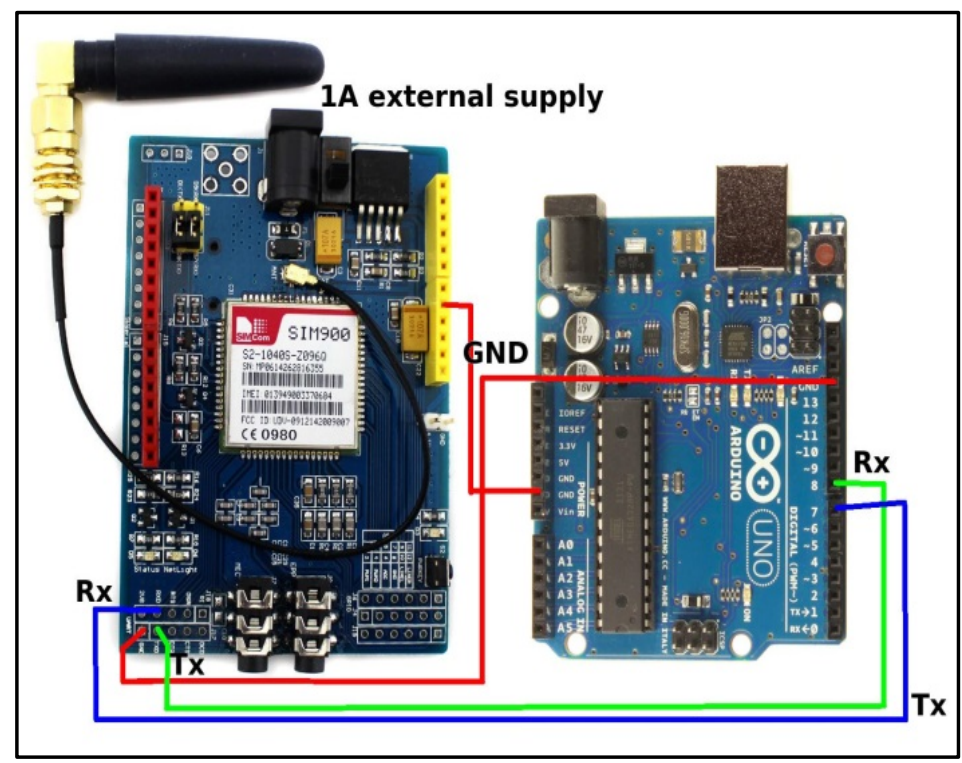

Fig. 8. Board of WEMOS-UNO GSM Shield

Table 1. GSM Module General Specs

\begin{tabular}{|l|l|}
\hline The Size of PCB & "71.4mm X 66.0mm X 1.6mm" \\
\hline Parameters & "PWR, status LED, net LED" \\
\hline "Power-Supply" & "12 V" \\
\hline The Protocol of the Communication & "UART" \\
\hline RoHS & "Yes" \\
\hline
\end{tabular}


Table 2. General measurements for vital human signals.

\begin{tabular}{|l|c|c|c|c|c|}
\hline \multirow{2}{*}{$\begin{array}{c}\text { Patients } \\
\text { Name }\end{array}$} & \multirow{2}{*}{ Age } & \multirow{2}{*}{\begin{tabular}{c} 
Gender \\
\cline { 5 - 6 }
\end{tabular}} & & \multirow{2}{*}{$\begin{array}{c}\text { Tale/ } \\
\text { The Suggested Model Pulse } \\
\text { Sensor (SEN -11574) }\end{array}$} & $\begin{array}{c}\text { Ground Truth Heartrate Surveillance } \\
\text { \& Body Temperature }\end{array}$ \\
\cline { 6 - 7 } & & Female & & Heart rate & Temperature \\
\hline Person 1 & 25 & Female & 92 & 90 & 36.2 \\
\hline Person 2 & 24 & Female & 84 & 72 & 36.5 \\
\hline Person 3 & 50 & Male & 73 & 65 & 37 \\
\hline Person 4 & 34 & Male & 67 & 85 & 36.5 \\
\hline Person 5 & 17 & Female & 82 & 82 & 36.9 \\
\hline Person 6 & 16 & Female & 85 & 82 & 36.4 \\
\hline Person 7 & 23 & Female & 82 & 80 & 36.5 \\
\hline Person 8 & 21 & Male & 78 & 75 & 37 \\
\hline Person 9 & 20 & Male & 82 & & 36.3 \\
\hline Person 10 & 39 & Female & 74 & & \\
\hline
\end{tabular}

Table 3. The outcome of body temperature data information

\begin{tabular}{|c|l|c|c|c|}
\hline \multirow{2}{*}{ No. } & \multirow{2}{*}{ Name } & \multicolumn{2}{|c|}{ Body Temperature (C) } & \multirow{2}{*}{ Error Rate } \\
\cline { 3 - 5 } & & DS18b20 Sensor & Digital Thermometer & \\
\hline 1. & Person 1 & 36.2 & 36.1 & $0.27 \%$ \\
\hline 2. & Person 2 & 36.5 & 36.4 & $0.27 \%$ \\
\hline 3. & Person 3 & 37 & 36.8 & $0.54 \%$ \\
\hline 4. & Person 4 & 36.5 & 36.5 & $0 \%$ \\
\hline 5. & Person 5 & 36.9 & 36.8 & $0.27 \%$ \\
\hline 6. & Person 6 & 36.4 & 36.4 & $0 \%$ \\
\hline 7. & Person 7 & 36.5 & 36.3 & $0.55 \%$ \\
\hline 8. & Person 8 & 36.8 & 36.7 & $0.27 \%$ \\
\hline 9. & Person 9 & 37 & 36.8 & $0.54 \%$ \\
\hline 10. & Person 10 & 36.3 & 36.3 & $0 \%$ \\
\hline & \multicolumn{2}{|c}{ Average error } & & $0.27 \%$ \\
\hline
\end{tabular}

Below, the screen samples of various vital signals readings utilized by the suggested system. 


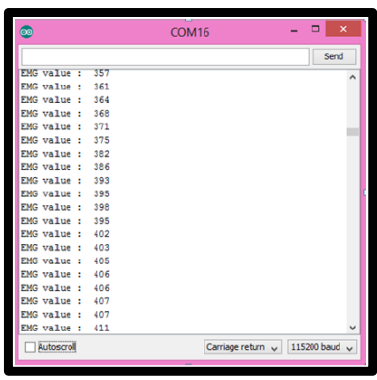

a) EMG Data

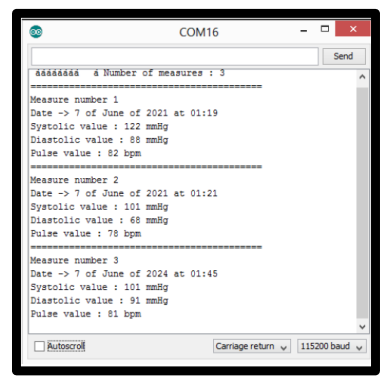

b) Blood Pressure Data

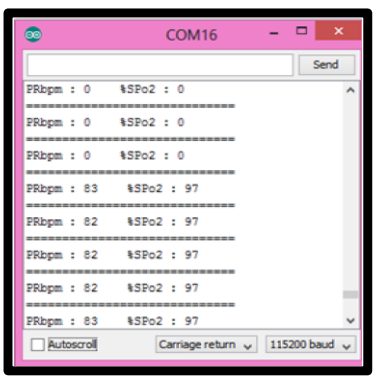

c) Pulse Oxymeter Data

Fig. 9.

\section{Conclusion}

A Wireless Health Caregiver surveillance system based on IoMTs has been designed, simulated and implemented that can wirelessly surveillance vital signs such as patient temperature and real-time pulse rate and immediately inform the medical staff in the event of emergencies. The system measures the health status of patients, such as pulse rate and temperature; if any of these parameters surpass predetermined critical values, the RF antenna module transmits the parameters to predefined telephone number in the form of SMS via a GSM module and shows the information for updating in the LCD specialists.

The method incorporates two widely utilized technologies to meet the job targets, namely Global Method for Mobile (GSM) and Radio Frequency technology. All the information gained from the human body via sensors is transmitted as digital values to the microcontroller system. At the transmitter and receiver sides, the values gained from the pulse rate and temperature sensors are also showed in alphanumerical form on the LCD. With global aging, electronic medical monitoring is expected to improve the worldwide healthcare system.

\section{References}

[1] A, S. et al., (2012)," Automatic Patient Monitoring System Utilizing Scatternet for Critical Healthcare". Journal of Theoretical and Applied Information Technology, 62-66.

[2] A.I, et al., (2001)," Real time ECG Transmission via Internet for Non Clinical Applications". Transactions on Biomedical Engineering, 253-257.

[3] Al-Rousan, A.-A. a. (2004)," Java based home automation system". IEEE Transactions on Consumer Electronics, 57-68. https://doi.org/10.1109/tce.2004.1309414 
[4] Abdulbaqi, A.S. et al., (2019), "Recruitment Internet of Things for Medical Condition Assessment: Electrocardiogram Signal Surveillance," REVISTA AUS 261/Doi:10.4206/aus.2019.n26.2.n26.2.52.

[5] Fatusi, et al., (2013), "Health Sector Reforms and Poverty". Ife: University of Ife Press.

[6] Shihab A. Hameed, V. M. (2008)," An Efficient Emergency Healthcare and Medical Information System", International Journal of Biometrics and Bioinformatics, 1-9.

[7] A. I. Heernandez et al.,"Real-time ECG transmission via Internet for nonclinical applications", IEEE Transaction on information technology in biomedicine, vol. 5, no. 3, pp. 253257, Sept. 2001. https://doi.org/10.1109/4233.945297

[8] Raised et al., (2005), "Bluetooth telemedicine processor for multichannel bio-medical signal transmission via mobile cellular networks". Information Technology in Biomedicine, IEEE Transactions on 9(1), 35. https://doi.org/10.1109/titb.2004.840070

[9] Abdulbaqi, A.S. and Ismail Yusuf Panessai, "Efficient EEG Data Compression and Transmission Algorithm for Telemedicine", Journal of Theoretical and Applied Information Technology (JATIT), Feb. (2019), Vol.97. No 4.

[10] Virone, et al., (2006, April),"An Advanced Wireless Sensor Network for Health Monitoring," In Trans disciplinary conference on distributed diagnosis and home healthcare (D2H2) (pp. 2-4).

[11] Hasiang Lin and I.C.J. (2004)," A Wireless PDA based Physiological Monitoring System for Patient Transport". IEEE Transactions on Information Technology in Biomedicine, 439447.

[12] M.F.A, R., \& .B, A.W. (2014)," A Wireless Emergency Telemedicine System for Patients Monitoring and Diagnosis". International Journal of Telemedicine and and Applications, 371-380.

[13] Purnima et al. (2014)," Zigbee and GSM based Health Monitoring System". International Journal of Advanced Research in Electrical, Electronics and Instrumentation Engineering, 6664-6669.

[14] U. A. et al., (2005)," A Wearable Multi parameter Medical Monitoring Alert System". Information Technology in Biomedicine, 415-427.

[15] Vilem S., M. P. (2010)," Embedded System Design For Health Supervisory Systems". Ubiquitos Computing and Communication Journal, 53-59.

[16] Abdulbaqi, A.S. et al., "Robust multichannel EEG Signals Compression Model Based on Hybridization Technique", International Journal of Engineering \& Technology, 7 (4), (2018) 3402-3405.

[17] Uttara Gogate et al., "Android Based Health Monitoring System for Cardiac Patients", International Research Journal of Engineering and Technology (IRJET), Vol. 04, Apr, (2017).

[18] Deiaa E. et al., "ECG Signal Transmissions Performance Over Wearable Wireless Sensor Networks", International Conference on Communication, Management and Information Technology (ICCMIT), Procedia Computer Science 65,(2015) 412 - 421. https://doi.org/10. 1016/i.procs.2015.09.109

[19] M. M. et al., "Compression of ECG signals based on DWT and exploiting the correlation between ECG signal samples," Int'1 J. of Communications, Network and System Sciences, vol. 2014, 2014.

[20] T. M. et al., "Assurance of energy efficiency and data security for ECG transmission in Ban's". IEEE transaction on biomedical engineering, Vol. 59, No.4, April, (2012).

[21] Lee, S. et al., (2011), "A real-time ECG data compression and transmission algorithm for an e-health device", IEEE Transactions on Biomedical Engineering, 58(9), 2448-2455. https:// doi.org/10.1109/tbme.2011.2156794

[22] Zhang, Y. et al., (2015)," Remote mobile health monitoring system based on smart phone and browser/server structure", Journal of healthcare engineering, 6(4), 717-738. https://doi. org/10.1260/2040-2295.6.4.717 
[23] Edward, H. et al., (2005)," A Wireless Health Monitoring System". International Conference on Information Acquisition (pp. 247-252). Hong Kong: Stony Brook Publisher.

[24] Maghawry, N. E., \& Ghoniemy, S. (2019). A Proposed Internet of Everything Framework for Disease Prediction. International Journal of Online \& Biomedical Engineering, 15(4). https://doi.org/10.3991/ijoe.v15i04.9834

[25] Thangarasu, G., \& Subramanian, K. (2019). Big data analytics for improved care delivery in the healthcare industry.

[26] Miskovic, V., \& Babic, D. (2016). Pervasive Personal Healthcare Service Designed as Mobile Social Network. International Journal of Interactive Mobile Technologies, 10(4). https:// doi.org/10.3991/ijim.v10i4.5913

\section{$9 \quad$ Authors}

Azmi Shawkat Abdulbaqi, University of Anbar, College of Computer Science \& Information Technology, Iraq, azmi_msc@uoanbar.edu.iq

Ahmed J.Obaid, Faculty of Computer Science and Mathematics, University of Kufa, Iraq, ahmedj.aljanaby@uokufa.edu.iq

Sundos Abdulameer Hmeed Alazawi, Department of Computer Science, Al Mustansiriyah University, Baghdad,Iraq, ss.aa.cs@,uomustansiriyah.edu.iq

Article submitted 2021-03-09. Resubmitted 2021-04-23. Final acceptance 2021-05-05. Final version published as submitted by the authors. 